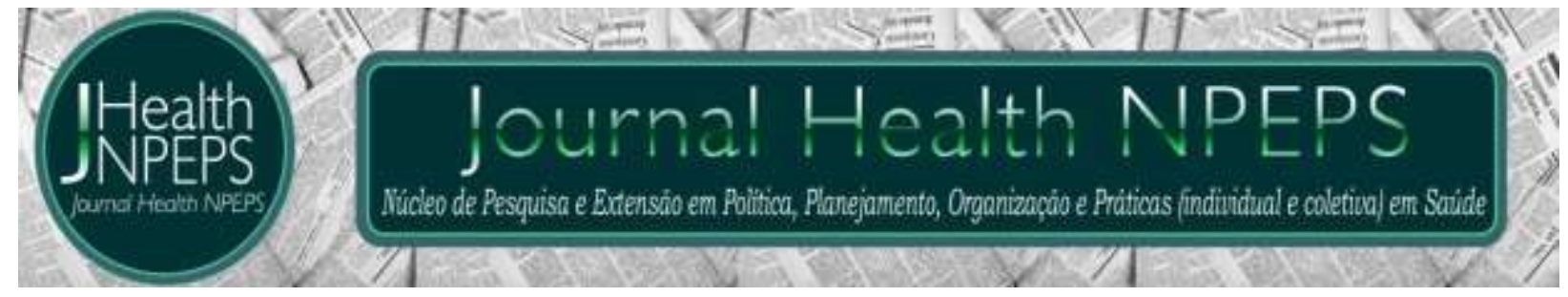

ARTIGO ORIGINAL

\title{
INFLUENCIAS URBANAS Y LA EXPOSICIÓN DE DROGAS EN FACEBOOK CON EL CONSUMO DE DROGAS
}

\section{URBAN INFLUENCES AND DRUG EXPOSURE ON FACEBOOK WITH DRUG USE}

\author{
INFLUÊNCIAS URBANAS E EXPOSIÇÃO DE DROGAS NO FACEBOOK COM CONSUMO DE \\ DROGAS
}

\author{
Jose Ignacio Vargas Martinez ${ }^{1}$, Francisco Rafael Guzman Facundo², Fabiola Peña \\ Cardenas $^{3}$, Maria Magdalena Alonso Castillo ${ }^{4}$, Karla Selene Lopez Garcia ${ }^{5}$
}

\begin{abstract}
RESUMEN
Objetivo: mostrar asociación del las características del barrio y la exposición de contenidos de drogas en Facebook con el consumo de drogas. Método: el diseño fue descriptivo correlacional, una muestra de 375 jóvenes con edades de 20 a 26 años, en una universidad pública. Se utilizó una cedula de datos sociodemográficos y los cuestionarios características del entorno de barrio, el uso de Facebook. Resultados: muestran que los jóvenes perciben un barrio con abuso de drogas, peligroso y personas con mala influencia para sus hijos. $30 \%$ han consumido drogas, prevaleciendo el mayor consumo en hombres (37.3\%), mujeres (24.4\%) alguna vez en la vida. Los días de uso de Facebook fueron $6.6(D E=1.0)$ semanalmente, una Mdn de $5.7(D E=4.4)$ y de 27.2 $(D E=6.2)$ al mes, el mayor dispositivo usado fue el teléfono (84.5\%). 30\% consumió alguna droga en la vida. Se observo una correlación positiva en las características del barrio y el uso de drogas $\left(x^{2}=4.59, p=.032\right)$ y para la exposición e invitación a través del Facebook donde hay drogas para su uso $(U=12490.5, p=.020)$. Conclusión: la exposición de contendidos de drogas en Facebook y las características negativas del barrio se relaciona con el uso de drogas.

Descriptores: Red social; Consumidores de Drogas; Adulto Joven.
\end{abstract}

\section{ABSTRACT}

Objective: show association of neighborhood characteristics and exposure of drug content on Facebook with drug use. Method: the design was descriptive correlational, a sample of 375 young people aged 20 to 26 years, in a public university. We used a sociodemographic data record and the questionnaires characteristic of the neighborhood environment, the use of Facebook. Results: Show that young people

\footnotetext{
${ }^{1}$ Profesor de Tiempo Completo. Cuerpo Academico Piscologia y Prevencion de Riesgo. Universidad Autónoma de Tamaulipas. Unidad Académica Multidisciplinaria Matamoros-UAT. México. E-mail: jvargas@docentes.uat.edu.mx Autor principal - Dirección de envío: Matamoros S/N, Zona Centro, 87000 Cd Victoria, Tamps., México.

${ }^{2}$ Doctorado en Ciencias de Enfermería Psiquiátrica. Universidad Autónoma de Nuevo Leon. México. E-mail: pako2001@hotmail.com

${ }^{3}$ Profesora e investigadora de tiempo completo de la licenciatura en Psicología. Universidad Autónoma de Tamaulipas. Unidad Academica MUtidsciplinaria Matamors-UAT. E-mail: fcardenas@docentes.uat.edu.mx

${ }^{4}$ Doctorado en Filosofía con énfasis en Psicología. lider del cuerpo Academico en Adicciones. Universidad Autónoma de Nuevo Leon. México. E-mail: magdalena_alonso@hotmail.com

${ }^{5}$ Doctor en Enfermería. Universidad Autónoma de Nuevo Leon. México. E-mail: karla.lopezga@uanl.edu.mx
} 
perceive a neighborhood with drug abuse, dangerous and people with bad influence for their children. 30\% have used drugs, with the highest consumption in men (37.3\%), women (24.4\%) at some point in life. The days of use of Facebook were 6.6 (SD = 1.0) weekly, an Mdn of 5.7 (SD = 4.4) and $27.2(S D=6.2)$ a month, the largest device used was the telephone (84.5\%). 30\% used some drugs in their lives. A positive correlation was observed in neighborhood characteristics and drug use $(x 2=4.59, p=.032)$ and for exposure and invitation through Facebook where there are drugs for use $(U=12490.5, p$ $=.020$ ). Conclusion: the exposure of drug content on Facebook and the negative characteristics of the neighborhood is related to drug use.

Descriptors: Social Networking; Drug Users; Young Adult.

\section{RESUMO}

Objetivo: mostrar associação das características do bairro, exposição de conteúdo de drogas no Facebook com consumo de drogas. Método: 0 desenho foi descritivo correlacional, numa amostra de 375 jovens com idade de 20 a 26 anos em uma universidade publica. Utilizou-se um questionário de dados sociodemográficos e os questionários características do entorno do bairro, o uso do Facebook. Resultados: mostram que os jovens percebem um bairro com abuso de drogas $(23,2 \%)$, perigoso $(12,5 \%), 31,2 \%$ pessoas com má influência para os seus filhos. $30 \%$ têm consumido drogas, maior consumo em homens $(37,3 \%)$, mulheres $(24,4 \%)$ alguma vez na vida. Os dias de uso de Facebook eram de 6,6\% ( $D E=1.0)$ semanal, uma Mdn DE 5,7\% (de=4.4), $27,2 \%(D E=6.2)$ ao mês, telefone maus utilizado para acessar $(84,5 \%) .30 \%$ consumem alguma droga na vida. Encontrou-se correlação positiva nas características de bairro e uso de drogas $\left(x^{2}=4.59, p=.032\right)$, exposição, convite do Facebook e drogas para consumo $(U=12490.5, p=.020)$. Conclusão: os conteúdos de drogas no Facebook e características negativas do bairro relacionam-se com consumo de drogas.

Descritores: Rede social; Usuários de Drogas; Jovem Adulto.

\section{INTRODUCCIÓN}

El uso de drogas ilícitas demanda una gran atención de los sistemas de salud pública ya que han provocado la muerte de aproximadamente 200 mil personas en el mundo, a menudo en edades productivas ${ }^{1}$. El panorama en México no difiere con lo antes señalado, ya que de acuerdo al Centro Nacional para la Prevención y el Control de Adicciones $^{2}$, de un total de 18,724 defunciones, 4,562 (24.4\%) ocurrieron bajo la influencia de alguna droga, a través del uso de armas de fuego (33.3\%), accidentes de tránsito (17.2\%) y asfixia (10.2\%).

Las tendencias globales registran en la región norte de México una mayor prevalencia de consumo de drogas ilegales (2.3\%), en comparación con la región centro (1.3\%) y sur (1.1\%). Las drogas de mayor consumo en el último año son la mariguana (1.2\%), seguida de la cocaína (.5\%), inhalantes (.1\%) y alucinógenos (.1\%). En cuanto a las variaciones en la edad de inicio del consumo de drogas ha disminuido, el promedio 
en el año 2002 era a los 23.6 años de edad mientras que en la última encuesta fue de 18.8 años, los hombres se inician casi dos años antes que las mujeres a consumir droga ilícitas ${ }^{2}$. Así mismo en grupos de jóvenes de 18 a 29 años han reportado consumo de drogas del $11 \%$ al $15 \%{ }^{3}$.

Los datos anteriores muestran que el sector juvenil es uno de los más afectado para el inicio del consumo de drogas ilícitas por lo que los jóvenes son considerados dentro de las poblaciones vulnerables para involucrarse con problemas relacionados con el consumo de drogas, ya que la juventud es una etapa de definiciones a nivel afectivo, sexual, social, intelectual y físico. La literatura muestra que las características del entorno social de los barrios son cada vez más reconocidas como estímulos importantes que influyen en la salud de los jóvenes ${ }^{4-5}$, en este sentido se ha evidenciado que las características del barrio en términos de la percepción del desorden o trastornos, así como aspectos socioeconómicos en el barrio donde se reside se asocia con el consumo de $\operatorname{drogas}^{6-8}$.

El barrio en México es considerado como la parte de una población de extensión relativamente grande, que contiene un agrupamiento social espontáneo y que tiene un carácter peculiar, físico, social, económico o étnico por el que se identifica a los miembros. Existe cierta evidencia que sugiere algunos aspectos del entorno cultural puede promover el inicio de consumo de alcohol y drogas en jóvenes. Es decir, los barrios desfavorecidos pueden proporcionar el contexto normativo en el consumo excesivo de alcohol donde no es sancionado comparado con otros barrios. En este sentido características como inseguridad y la falta de apoyo entre los miembros pueden favorecer el consumo de alcohol y drogas ${ }^{9}$. En este sentido para este estudio se conceptualiza las características del barrio como la percepción que los jóvenes tienen sobre el lugar donde residen en términos particulares de la comunidad tales como seguridad, aspectos de organización entre vecinos.

Aunado a lo anterior otro aspecto contextual que pude potencializar la vulnerabilidad para el consumo de drogas es que los jóvenes actuales se caracterizan por interactúan más por redes sociales sin tener contacto personal, los sitios de redes sociales como el Facebook proporcionan un enfoque hacia la identificación y comunicación, donde los teléfonos inteligentes y tabletas son herramientas principales de comunicación entre los jóvenes. Para el 2013 Facebook llegó a los 1230 millones de usuarios. Actualmente Facebook ofrece una variedad de servicios a los usuarios, se 
pueden intercambiar fotos o mensajes, para ello, el servidor de Facebook posee herramientas de búsqueda y de sugerencia de amigos. En los muros de cada perfil de usuario permite que los amigos escriban mensajes para que el usuario los vea. Así mismo el usuario puede publicar fotografías e imágenes dentro de su perfil y otros perfiles.

La Asociación Mexicana de Internet ${ }^{10}$ reporta en su primer estudio sobre redes sociales en México, que el número de usuarios alcanzó los 34.9 millones. Dentro de las redes sociales más utilizadas es el Facebook, estima que el tiempo promedio de conexión diario es de cinco horas, el acceder a las redes sociales es una de las principales actividades en línea con el $82 \%$. Así mismo la literatura reciente ha identificado el uso de redes sociales electrónicas como un posible factor de riesgo para el inicio de consumo de drogas, se ha identificado que la exposición de contenidos de alcohol en Facebook y drogas se asocia con el consumo de drogas en adolescentes y jóvenes ${ }^{11-14}$.

Aunado a todo lo anterior la ciudad de Matamoros, es una ciudad en el noreste del estado de Tamaulipas, en México, colinda con la ciudad fronteriza de Brownsville, Texas. Esta ciudad se ha caracterizado por los altos índices de inseguridad atribuida a bandas del crimen organizado. Como se refirió anteriormente la literatura muestra que las características del entorno social de los barrios son cada vez más reconocidas como estímulos importantes que influyen en la salud de los jóvenes, es posible que los jóvenes residentes de estas zonas perciban de forma diferente las características de sus barrios y esté relacionado con conductas no saludables como el consumo de drogas ${ }^{4-8}$. Cabe señalar que en el estudio de Hill y Ángel ${ }^{9}$ reportan que vivir en un barrio caracterizado por problemas con las drogas, la delincuencia, el desempleo, inactividad juvenil, casas abandonadas y con escasa seguridad pública puede ser psicológicamente angustiante y pueden ser determinantes para el consumo de alcohol y drogas.

Aunado a todo lo anterior existe escasa evidencia que integren estímulos contextuales para explicar el consumo drogas en jóvenes de zonas fronterizas, por experiencia empírica del investigador del estudio se sabe que el obtener drogas es más accesible por la cercanía de los Estados Unidos y el fenómeno de oferta de drogas es mucho más evidente que en otros estados de México. Esta situación permite reflexionar sobre la necesidad de abordar el fenómeno del consumo drogas en jóvenes de zonas fronterizas. 
Por tal razón es importante explorar en jóvenes de la frontera norte si las características de su barrio, así como la exposición de contenidos de drogas en Facebook puede ser estímulos que favorezca el consumo drogas, el cuidado en la prevención del consumo de drogas debe estar dirigido al control de los estímulos, ya que al integrar en la prevención del consumo de drogas la evaluación de estímulos contextuales, podría contribuir a optimizar la respuesta adaptativa del joven hacia el no consumo drogas. El cuidado preventivo es la principal estrategia en el fenómeno de las drogas ya que es conocido que la prevención es una herramienta efectiva y económica. Por lo anterior se pretende realizar un estudio con el objetivo mostrar la asociación del las características del barrio y la exposición de contenidos de drogas en facebook con el consumo de drogas.

\section{MÉTODOS}

El estudio fue descriptivo correlacional ${ }^{15}$. La población se conformaron de 2,726 jóvenes inscritos en una universidad pública de la Ciudad de Matamoros, Tamaulipas. El diseño de muestreo fue aleatorio estratificado con asignación proporcional al estrato, la carrera forma el estrato, se formaron cinco estratos (Enfermería, Psicología, Seguridad, Medicina y Sistemas computacionales), dentro de cada estrato se realizó un muestreo por conglomerados unietápico utilizando los grupos como conglomerados. El tamaño de la muestra se calculó por medio del software nQueryAdvisor $4.0^{16}$, tomando en cuenta como parámetros un Modelo de Regresión lineal, nivel de significancia de .05, potencia de 90\%, con un Coeficiente de correlación de .09 considerado un tamaño de efecto mediano ${ }^{17}$, considerando una tasa de no respuesta de $10 \%$ y efecto de diseño de 1.5, dando una muestra de 375 sujetos definidos en 15 grupos.

Para la recolección de los datos se utilizó una cedula de datos sociodemográficos, así como tres cuestionarios que miden Características del Entorno de Barrio $^{18}$ (Neighborhood Short Form el Uso de Facebook ${ }^{19}$. La Cédula de Datos Personales y Prevalencias de Consumo de Alcohol y Drogas (CDPPCAD) recaudó información sobre datos biológicos (edad y sexo) y sociodemográficos (ocupación, personas con la que vive, semestre y residencia actual). Así mismo se obtendrán prevalencias de consumo de drogas alguna vez en la vida, en el último año, último mes y en los últimos siete días; además de la edad de inicio. 
La forma corta del instrumento que mide las características del entorno del Barrio $^{18}$ (Neighborhood Short Form), permitió valorar la percepción que tienen los jóvenes sobre algunas características del barrio como apoyo social, la seguridad del barrio y orgullo por el barrio. Este instrumento fue desarrollado por Martínez ${ }^{18}$ basado en la revisión de la literatura. Este instrumento cuenta con 9 preguntas, el cual es autoaplicable, con un tiempo aproximado de cinco minutos, cada pregunta tiene cuatro opciones de respuestas que van desde 1 (muy parecido a mi barrio) a 4 (no como mi barrio). Cuenta con tres subescalas, seguridad (preguntas 3, 6 y 9), apoyo social (1, 4 y 7,) y orgullo (2, 5 y 8). La puntuación total es de 36, a mayor puntuación indica menor grado de calidad en las características de la colonia. No se encontró evidencia sobre su aplicación en población mexicana. En población residente en Estados Unidos ha mostrado una confiabilidad aceptada para la escala general ( $a=.87)$ y para cada una de las subescalas (seguridad [a=.85]; apoyo social [a=.79] y orgullo por el barrio $[a=.73])$.Para fines de este estudio se realizó por primera una adaptación transcultural para el idioma español a través del método traducción-retraducción con profesionales bilingües de la Universidad Autónoma de Tamaulipas con el objetivo de buscar su equivalencia a nivel semántico, conceptual, de contenido, técnico y de criterio para el idioma español. Posteriormente se realizó una prueba piloto para medir las propiedades psicométricas, el instrumento obtuvo un Alpha de Cronbach.

El Cuestionario de Uso de Facebook $^{19}$ (CUF) fue utilizado para medir la exposición de contenidos de drogas en Facebook, desarrollado por Crow ${ }^{19}$, este instrumento ha sido aplicado en adolescentes de Estados Unidos y Mexicanos, el instrumento mide los contenidos relacionados con el alcohol o drogas en Facebook, es un cuestionario autoaplicable que consta de 15 preguntas que cuantifica la frecuencia y datos generales de uso de Facebook y la exposición sobre contenidos relacionados con el alcohol o drogas en Facebook. Las preguntas de la 1 a la 3 miden el uso de Facebook en término de días que se utiliza Facebook en el último mes y semana, así como también el promedio de horas utilizado en el día. De la pregunta 4 a la 10 exploran la exposición a contenidos relacionados con el consumo de drogas en Facebook. Finalmente, las preguntas de la 11 a la 15 obtienen datos generales sobre el uso de esta red social. Cada pregunta corresponde a una variable continua y para el apartado de exposición a contenidos relacionados con el consumo de drogas en Facebook se realizaron índices que obtuvieron valores de 0 a 100 de tal manera que a mayor puntuación mayor es la 
exposición de consumo de drogas en Facebook.

Navarro ${ }^{20}$ llevó a cabo la validación de este instrumento por medio un panel de expertos, así mismo se realizó la validez de contenido mostrando buena comprensión del instrumento mostrando una confiabilidad aceptable para el cuestionario en general ( $a=.75)$ y para las subescalas de frecuencia de uso $(a=.74)$ y exposición de contenidos relacionados con el alcohol $(a=.76)$. Cabe destacar que en una prueba piloto obtuvo una consistencia interna a través de la aplicación del Alpha de Cronbach de .86.

Después de obtener la aprobación del estudio por las Comisiones de Ética e Investigación de la Facultad de Enfermería de la Universidad Autónoma de Nuevo León, se procedió a solicitar autorización por escrito a autoridades de las Instituciones Educativas para realizar la investigación. A los jóvenes seleccionados se les buscó en cada aula de clase en coordinación con personal de las instituciones educativas y se les citó en aula asignada por las autoridades o en su caso en la misma aula donde toman su clase previo permiso del maestro de la materia. Una vez reunidos se procedió a explicar de forma clara y sencilla a cada grupo, los objetivos de la investigación, la dinámica de los instrumentos de recolección, el tiempo requerido, y se resolvieron dudas que tuvieron los jóvenes. Posteriormente se les solicitó su consentimiento informado. Cabe destacar que se cuidó que se realizara en horarios que no interfirieran con actividades escolares programadas. El llenado de los cuestionarios se estimó en un tiempo entre 20 minutos, al finalizar el llenado de los cuestionarios los participantes entregaran los cuestionarios a las personas de apoyo quienes fueron capacitados para administrar los instrumentos y despejar dudas en caso de que se presentaran, finalmente se les agradeció a cada uno de los jóvenes por su participación. Cabe destacar que en todo momento el presente estudio se apegó a lo dispuesto en el Reglamento de la Ley General de Salud en materia de Investigación para la Salud ${ }^{21}$.

Para el procesamiento de los datos se utilizó el paquete estadísticos SPSS ${ }^{\circledR}$ versión $20.0^{22}$ para Windows. Se utilizó la estadística descriptiva e inferencial. El análisis descriptivo se realizara a través de frecuencias, proporciones, medidas de tendencia central y de variabilidad, esto para describir la población del estudio y las variables utilizadas. Por otro lado, se determinó la consistencia interna de los instrumentos mediante el Coeficiente Alpha de Cronbach. Previo al análisis se procedió a calcular sumatorias, promedios e índices de los instrumentos. Para identificar las relaciones entre las variables del estudio, se utilizó Chi cuadrado y $U$ de Mann Whiney. 


\section{RESULTADOS}

Respecto algunas características sociodemográficas de la muestra se destaca un promedio de edad de 20.26 años (DE=1.73), 58\% son mujeres, $43 \%$ estudia médico cirujano, $16 \%$ psicología, $15.5 \%$ ingeniero en sistemas, $13 \%$ enfermería y $13 \%$ seguridad y medio ambiente, $21 \%$ mencionó estudiar y trabajar y la mayoría vive con ambos padres (60.3\%), $18 \%$ con la madre, solo $2.7 \%$ vive con el padre y $19 \%$ vive solo o con amigos.

En relación con algunas características del barrio en los jóvenes de la frontera norte $23.2 \%$ perciben que en su barrio existe abuso de drogas y alcohol, $12.5 \%$ consideran que su barrio es peligroso, $18.1 \%$ refiere que existen edificios y parques viejos y abandonados y $31.2 \%$ refiere que existen personas en su barrio que podrían ser mala influencia.

En base a la descripción de uso de Facebook, el promedio de los días de uso de Facebook por semana fue de $6.6(D E=1.0)$, respecto a las horas de uso de Facebook por día se observa una media de 5.7 ( $D E=4.4)$ y en relación a los días de uso en los últimos 30 días de 27.2 ( $D E=6.2)$. El dispositivos más frecuentes para acceder a Facebook, fue por medio de celulares (84.5\%), seguido de laptop (8.3\%).

En la tabla 1 se muestran el consumo de drogas en jóvenes de la frontera norte, cabe mencionar que las drogas de mayor consumo fueron marihuana, cocaína y drogas médicas. Se observa que cerca del 30\% de los jóvenes han consumido alguna droga ilícita al menos una vez en la vida. Por otro lado en la tabla 2 se muestra el consumo de estas drogas por género destacando diferencias significativas del consumo de cualquier droga, de consumo de mariguana y de cocaína por sexo, mostrando mayor prevalencia de consumo en los hombres que en las mujeres, no obstante en el último año y en el último mes el consumo no mostró diferencias significativas. Cabe destacar que el consumo de drogas médicas es similar entre hombres y mujeres.

Tabla 1 - Prevalencias de consumo de drogas ilícitas.

\begin{tabular}{lcccc}
\hline \multicolumn{1}{c}{ Prevalencia } & $n$ & $P$ & LI & IC 95\% \\
\hline Cualquier droga & & & 25 & 34 \\
Alguna vez en la vida & 112 & 29.9 & 11 & 19 \\
Último año & 58 & 15.5 & 3 & 7 \\
Último mes & 20 & 5.3 & 21 & 30 \\
\hline Mariguana & & & 7 & 14 \\
Alguna vez en la vida & 96 & 25.6 & 10.9 & 21 \\
Último año & 41 & 10.9 & \\
\hline
\end{tabular}




\begin{tabular}{lcccc}
\hline Último mes & 13 & 3.5 & 1 & 5 \\
\hline $\begin{array}{l}\text { Cocaína } \\
\text { Alguna vez en la vida }\end{array}$ & 29 & 7.7 & 5 & 10 \\
Último año & 12 & 3.2 & 1 & 4 \\
Último mes & 5 & 1.3 & 0 & 2 \\
\hline $\begin{array}{l}\text { Drogas medicas } \\
\text { Alguna vez en la vida }\end{array}$ & 16 & 4.3 & & \\
Último año & 9 & 2.4 & 2 & 4 \\
Último mes & 4 & 1.1 & 1 & 2
\end{tabular}

Nota: $n=$ muestra $P=$ tasa de prevalencia, $I C=$ Intervalo de Confianza, $L I=$ Límite Inferior, $L S$ = Límite Superior

Tabla 2 - Prevalencias de consumo de drogas ilícitas por género.

\begin{tabular}{|c|c|c|c|c|c|c|c|c|}
\hline \multirow{2}{*}{ Prevalencias } & \multicolumn{3}{|c|}{ Hombres } & \multicolumn{3}{|c|}{ Mujeres } & \multirow[b]{2}{*}{$x^{2}$} & \multirow[b]{2}{*}{$P$} \\
\hline & $f$ & $P$ & IC 95\% & $f$ & $P$ & IC 95\% & & \\
\hline \multicolumn{9}{|l|}{ Cualquier droga } \\
\hline Alguna vez en la vida & 59 & 37.3 & {$[29-44]$} & 53 & 24.4 & {$[18-30]$} & 7.28 & .007 \\
\hline Último año & 28 & 17.7 & {$[11-23]$} & 30 & 13.8 & {$[9-18]$} & 1.06 & .303 \\
\hline Último mes & 12 & 7.6 & {$[3-11]$} & 8 & 3.7 & {$[1-6]$} & 2.76 & .096 \\
\hline \multicolumn{9}{|l|}{ Marihuana } \\
\hline Alguna vez en la vida & 57 & 36.1 & [28-43] & 39 & 18.0 & {$[12-23]$} & 15.7 & .001 \\
\hline Último año & 22 & 13.9 & {$[8-19]$} & 19 & 8.8 & {$[4-12]$} & 2.50 & .113 \\
\hline Último mes & 7 & 4.4 & {$[1-7]$} & 6 & 2.8 & {$[0-4]$} & .758 & .384 \\
\hline \multicolumn{9}{|l|}{ Cocaína } \\
\hline Alguna vez en la vida & 17 & 10.8 & {$[6-15]$} & 12 & 5.5 & {$[2-8]$} & 3.50 & .006 \\
\hline Último año & 6 & 3.8 & {$[0-6]$} & 6 & 2.8 & {$[0-4]$} & .315 & .575 \\
\hline Último mes & 4 & 2.5 & {$[0-5]$} & 1 & 0.5 & {$[0-1]$} & 2.98 & .084 \\
\hline \multicolumn{9}{|l|}{ Drogas medicas } \\
\hline Alguna vez en la vida & 7 & 4.4 & {$[1-7]$} & 9 & 4.4 & {$[1-6]$} & .018 & .894 \\
\hline Último año & 3 & 1.9 & {$[0-4]$} & 6 & 2.8 & {$[0-4]$} & .293 & .588 \\
\hline Último mes & 2 & 1.3 & {$[0-3]$} & 2 & 0.9 & {$[0-2]$} & .103 & .749 \\
\hline
\end{tabular}

En la tabla 3 se muestra las características del barrio que se asocian con el consumo de drogas ilícitas, se destaca que las características que muestran asociación son que exista abuso de drogas en el barrio, que existan edificios o parques abandonados y que se perciba que haya personas que sean mala influencia.

Tabla 3 - Características del barrio que se asocian con el consumo de drogas.

\begin{tabular}{|c|c|c|c|c|}
\hline \multirow{2}{*}{ Características del barrio } & \multicolumn{2}{|c|}{ Consumo de drogas } & \multirow{2}{*}{$x^{2}$} & \multirow[b]{2}{*}{$p$} \\
\hline & $\mathrm{Si}$ & No & & \\
\hline \multicolumn{5}{|l|}{ Existe abuso de drogas } \\
\hline$S i$ & 30.4 & 20.2 & 4.59 & .032 \\
\hline No & 69.6 & 79.8 & & \\
\hline \multicolumn{5}{|c|}{ Existen edificios y parques abandonados } \\
\hline So & $\begin{array}{l}24.1 \\
75.9\end{array}$ & $\begin{array}{l}15.6 \\
84.4\end{array}$ & 3.83 & .050 \\
\hline \multicolumn{5}{|l|}{ Existen personas que podrían ser mala } \\
\hline $\begin{array}{ll}\text { influencia } & \mathrm{Si} \\
& \text { No }\end{array}$ & $\begin{array}{l}42.0 \\
58.0\end{array}$ & $\begin{array}{l}26.6 \\
73.4\end{array}$ & 8.62 & .003 \\
\hline
\end{tabular}

Nota: $x^{2}=$ Prueba de Chi Cuadrada de Person, $p=$ significancia estadística 
La tabla 4 muestra el contraste de tendencia central del índice de exposición de contenidos de alcohol en Facebook, así como las invitaciones a fiestas en Facebook donde se involucra drogas en los últimos 30 días entre el consumo de drogas ilícitas por la Prueba $U$ de Mann-Whitney. Los resultados muestran diferencias significativas destacando mayores índice de exposición de contenidos de drogas en Facebook y mayor numero de invitaciones a fiestas por Facebook donde se involucra el consumo de drogas en los jóvenes que han consumido drogas comparado con aquellos que nunca han consumido drogas ilícitas.

Tabla 4 - Contraste de tendencia central del la exposición de contenidos de drogas en Facebook e invitaciones a fiestas por Facebook donde se involucran drogas entre el consumo de drogas ilícitas por la Prueba U de Mann-Whitney.

\begin{tabular}{|c|c|c|c|c|c|c|}
\hline \multirow[t]{2}{*}{ Consumo de drogas } & \multicolumn{6}{|c|}{ Índice de exposición de contenidos de drogas en Facebook } \\
\hline & $n$ & $\overline{\bar{X}}$ & Mdn & $D E$ & $U$ & $p$ \\
\hline Si & 112 & 3.82 & 0.95 & 13.11 & 12490.5 & .020 \\
\hline \multirow[t]{2}{*}{ No } & 260 & 5.98 & 0 & 9.86 & & \\
\hline & \multicolumn{6}{|c|}{$\begin{array}{l}\text { Invitaciones a fiestas por Facebook donde se involucran drogas en } \\
\text { los últimos } 30 \text { días }\end{array}$} \\
\hline Si & 112 & 1.57 & 0 & 1.84 & 12445.0 & .001 \\
\hline No & 260 & 0.43 & 0 & 4.51 & & \\
\hline
\end{tabular}

\section{DISCUSIÓN}

Las necesidades básicas del ser humano por naturaleza es su sociabilidad, saberse parte de una comunidad, de influir en los demás, darse cuenta de lo que pasa en el entorno ${ }^{23}$. Las redes sociales tienen esta factibilidad, pero una en especial el Facebook el cual contiene los componentes necesarios para que los jóvenes puedan alcanzar esa sociabilidad entre ellos, como parte del entorno, el cual se ve influenciado por la era cibernética y por donde las conductas de estos se ven influenciadas para proceder de muchas formas, en su actuar, además de el lugar donde residen como son las características de su barrio, como parte de un complemento para compartir demasiadas situaciones de la vida o que estas puedan influir en riesgos de salud como lo es el consumo de drogas ilícitas.

Para el abuso de drogas ilícitas en el barrio se observa que el $23.2 \%$ de los encuestados tienen esta precepción de su barrio, dada las características en que se encuentra, así mismo se observo que el consumo de drogas ilícitas entre los jóvenes que 
se reúnen en las esquinas de sus barrio ${ }^{27-26}$, consumidores de drogas dentro del género no hay significancia estadística ya que tanto hombre como mujeres están consumiendo a la par, también observan que el tiempo que pasan consumiendo es entre 5 a 6 horas durante el día, esto puede concordar con la percepción de los habitantes para el abuso de drogas dentro del barrio, como también se observa que aunque sean trabajadores las personas consumen más drogas ilícitas y por más tiempo ${ }^{28}$.

Una discrepancia que existe en los resultados es como ellos tienen la precepción muy alta del uso de drogas de su barrio, además de sentirse orgulloso de él (38.4\%) y a pesar de esto no lo consideran como un barrio peligroso (30.1\%), esto puede deberse a que la población ya se está acostumbrando a la alta tasa de inseguridad que prevalece en esta región del norte del país, debido a los grupos delincuenciales o el observar el consumo de drogas ilícitas ya se vuelve tan común en su barrio.

Otro hallazgo es como consideran ellos la confianza que tienen en otras personas dentro de su barrio (35.5\%), pero al preguntarles si hay personas que pueden influir de forma negativa con sus niños (31.2\%), lo consideran muy parecido a su barrio, así mismo la literatura muestra como las precepciones de los habitantes dentro de su barrio o colonia con similares características los perciben de forma negativa para poder vivir o educar a sus hijos $28-26$.

Dentro del uso de Facebook y su influencia para el consumo de drogas ilícitas aquí hay que resaltar que no dentro de la literatura existente no se encontró algún estudio sobre el consumo de drogas ilícitas que los jóvenes reciben menos de una

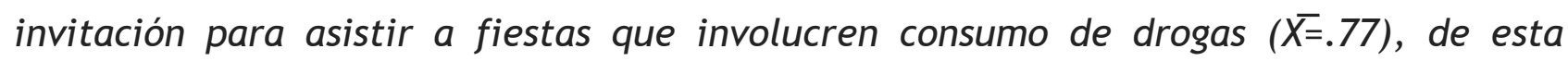
misma forma los jóvenes observan solo $\bar{X}=1.2$ de anuncios publicados en esta red social, dentro de los grupos de Facebook que involucren el consumo de drogas ilícitas solo observan dentro de los últimos 30 días un promedio de .99.

En lo que corresponde a la exposición de contenidos de drogas ilícitas en Facebook los jóvenes reciben un promedio de 12 exposiciones semestrales incluyendo fotografías y mensajes que involucren drogas ilícitas, para el apartado de figuras públicas (24.8\%) y de aplicaciones (11.8\%) involucrando drogas ilícitas, es una parte importante en tomar en cuenta ya que él los jóvenes tienen un promedio aproximado de más de 6 horas por día interactuando dentro de esta red social, así como una $\mathrm{Mdn}=7$ días semanales, lo que puede atraer comportamiento distintos y esto aumentar el consumo de drogas ilícitas en los jóvenes ya que esta red social Facebook conlleva a 
cambiar conductas dentro de ellos, ya sea por estar a la moda sentirse dentro de un contexto social igual que los demás amigos o por estar en un estado de ánimo muy relajado (fiestas, reuniones, festejos), dando una representación positiva para esta actitud del consumir drogas ilícitas. Facebook demuestra que la necesidad de ser sociable es la cualidad más importante del ser humano, mientras que la de obtener información queda en segundo lugar ${ }^{24}$. La tecnología estaba ahora al servicio de la principal cualidad humana la sociabilidad, también permite de manera indirecta conocer y estar al día en la información que ahí se produce intercambia ${ }^{23}$.

\section{CONCLUSIÓN}

Las principales aportaciones que este estudio realiza, son principalmente como la red social de Facebook está influyendo en los jóvenes aunque es una mínima parte en relación al consumo de drogas ya que solamente algunos jóvenes son los que llegan a postear fotos, texto e imágenes con este contenido de drogas, debido a la prohibición por parte de la legislación legal que conlleva, aun así es posible observar este tipo de posteos, dando una clara imagen de la conducta que algunos jóvenes tienen, por lo tanto no hay que subestimar las redes sociales sobre todo las que pueden ser vistas por muchas personas incluyendo a los niños.

Aunado a la influencia del barrio donde viven y con las características de ser barrios violentos o con influencia negativa por parte de algunos pobladores donde el consumo de alcohol y drogas puede ser algo visto "normal", por esto la trascendencia de explorar esta red social y su influencia con los jóvenes, para evitar el riesgo de esta influencia negativa, por lo tanto es importante continuar con estos estudios y variables, para futuros estudios como parte de intervenciones por el personal de enfermería que es una de las profesiones más cercanas a la población dentro de los centros comunitarios.

\section{REFERENCIAS}

1. United Nations Office on Drug and Crime. World Drug Report 2015 highlights evidence-based drug prevention and treatment. [Internet]. 2015 [acceso 20 de 
Septiembre de 2015]. Disponible en: https://www.unodc.org/unodc/es/drugprevention-and-treatment/index.html

2. Centro Nacional para la Prevención y el Control de Adicciones (CENADIC). Programa de Acción Específico, Prevención y Tratamiento de las Adicciones actualización 2011 - 2012. [Internet]. 2011 [acceso 19 de Agosto de 2014]. Disponible en: http: //www.cenadic.salud.gob.mx/PDFS/publicaciones/pae-2011.pdf.

3. Llamas M, Guzmán F, Rodríguez A \& Alonso C. Norma subjetiva e intención del consumo de marihuana en jóvenes universitarios de México. Cienc enferm. 2012; XVIII (1): 57-66.

4. Kim J. Neighborhood disadvantage and mental health: The role of neighborhood disorder and social relationships. Soc sci res. 2001; 39:260-71.

5. Reyes JC, Colón HM, Robles RR, Negrón J, Marrero CA, Matos TD, Calderón J, Pérez O. Alcohol use among adolescents in Puerto Rico: the influence of physical and social neighborhood disorder. Bol Asoc Med P R. 2006; (3), 186-91.

6. Matheson FI, White HL, Moineddin R, Dunn JR, Glazier RH. Drinking in context: the influence of gender and neighborhood deprivation on alcohol consumption. J epidemiol community health. 2012; 66(6):e4.

7. Jackson N, Denny S, Ameratunga S. Social and socio-demographic neighborhood effects on adolescent alcohol use: A systematic review of multi-level studies. Soc sci med. 2014; 115:10-20.

8. Shimotsu J, W, MacLehose N, Forster L. Neighborhood socioeconomic characteristics, the retail environment, and alcohol consumption: a multilevel analysis. Drug alcohol depend. 2013; 132(3):449-56.

9. Hill T, Angel R. Neighborhood Disorder, Psychological Distress, and Heavy drinking. Soc sci med. 2005; 61(5):965-75.

10. Asociación Mexicana de Internet. Estudio sobre los hábitos de los usuarios de internet en México 2014 [Internet]. 2014 [acceso 03 de Febrero de 2015]. Disponible en:https://www.amipci.org.mx/estudios/habitos_de_internet/Estudio_Habitos_del_ Internauta_Mexicano_2014_V_MD.pdf

11. Huang GC, Unger JB, Soto D, Fujimoto K, Pentz MA, Jordan-Marsh M, et al. Peer influences: the impact of online and offline friendship networks on adolescent smoking and alcohol use. J adolesc health. 2014; 54(5):508-14. 
12. Griffiths R, Casswell S. Intoxigenic digital spaces? Youth, social networking sites and alcohol marketing. Drug alcohol rev. 2010; 29:525-30.

13. Fournier AK, Clark SW. Do College Students use Facebook to Communicate about Alcohol? An Analysis of Student Profile Pages. Cyberpsychol: J Psychosoc res cyberspace. $2011 ; 5(2): 1-12$.

14. Moreno MA, D’Angelo J, Kavincsky LE, Kerr B, Zhang C, Eickoff J. Emergence and predictors of alcohol reference displays on Facebook during the first year of college. Comput human behav. 2014; 30:87-94.

15. Burns N, Grove SK. The practice of nursing research: appraisal, synthesis and generation of evidence. Missouri: Saunders; 2009.

16. Elashoff, JDD, Crede F. nQuery Advisor (Versión 7.0) [Software de computación]. Los Angeles: Statistical Solutions LTD; 2007.

17. Cohen J. Statistical Power Analysis for the Behavioral Sciences. Lawrence: Erlabaum Associates; 1988

18. Martinez LM. Neighborhood context and the development of African American children. New York: Garland; 2000.

19. Crow BM. Does Facebook impact college students' alcohol consumption? University Carbondale, Chicago, Illinois. [Dissertation]. University Carbondale, Chicago, Illinois; 2013

20. Navarro EIP, De Anda PJ, Gamez ME, Candia JS \& Guzmán FR. Exposición a contenidos de uso de alcohol en Facebook y consumo de alcohol en adolescentes. Rev enferm Herediana. 2016; 9(1):43-49.

21. Secretaría de Salud. Reglamento de la Ley General de Salud en materia de Investigación para la Salud 1987. [Internet]. [acceso 19 de Agosto del 2015]. Recuperado de: http://www.salud.gob.mx/unidades/ cdi/nom/compi/rlgsmis.html

22. IBM $®$ SPSS $®$ Statistics for Windows (Versión 20.0.0) [Software de computación]. Armonk, NY: IBM Corp.

23. El-Sahili LF. Piscología del Facebook. Guanajuato, México, ed. Universidad de Guanajuato; 2014.

24. Reig $D$ \& Vilchez LF. Los jóvenes en la era de la hiperconectividad: tendencias claves y miradas. Madrid: Fundación Telefónica y Fundación Encuentro; 2013. 
25. Furr-Holden $\mathrm{C}$, Lee $M$, Milam A, Johnson $\mathrm{R}$, Lee $\mathrm{K}$, lalongo $\mathrm{N}$. The Growth of Neighborhood Disorder and Marijuana Use Among Urban Adolescents: A Case for Policy and Environmental Interventions. J stud alcohol drugs. 2011; 72: 371-379.

26. Duncan D, Palamar J, Williams J. Perceived neighborhood illicit drug selling, peer illicit drug disapproval and illicit drug use among U.S. high school seniors. Subst abuse treat prev policy. 2014; 9:35.

27. Rossi D, Singh D, Pawlowicz M, Touzé G, Bolyard M, Mateu-Gelabert P, et al. Changes in time-use and drug use by young adults in poor neighborhoods of Greater Buenos Aires, Argentina, after the political transitions of 2001-2002: Results of a survey. Harm reduc j. 2011; 8:2.

28. Tucker JS, Pollard MS, de la Haye K, Kennedy DP, Green HD Jr. Neighborhood Characteristics and the Initiation of Marijuana Use and Binge Drinking. Drug alcohol depend. 2013; 128(1-2):83-9.

Conflito de interesses: Os autores declaram não haver conflito de interesses.

Como citar este artigo: Martinez JIV, Facundo FRG, Cardenas FP, Castillo MMA, Garcia KSL. Influencias urbanas y la exposición de drogas en facebook con el consumo de drogas. Journal Health NPEPS. 2017; 2(1):161175. 